\title{
A TWO-PARAMETER THEORY FOR VENUS SPECTRA
}

\author{
JOSEPH W. CHAMBERLAIN \\ Planetary Sciences Division, Kitt Peak National Observatory, ${ }^{*}$ Tucson, Ariz., U.S.A.
}

\begin{abstract}
An approximate, analytic theory has been developed for the formation of spectral absorption lines in a hazy atmosphere that scatters isotropically and has a homogeneous mixture of scattering and absorbing matter. The behavior - i.e., the curve of growth and the dependence of equivalent width on incident and emergent angles - has been examined for various possible situations, with emphasis on the physical reasons for a particular behavior. It is emphasized that two ratios - essentially the line and continuum absorption coefficients relative to the scattering coefficient - are important in any quantitative theory of the curve of growth and phase variation of the absorption spectrum.
\end{abstract}

In a semi-infinite atmosphere, categories of absorption lines are fixed by two fundamental parameters, whose relative importance has not previously been clearly distinguished: The probability of photon absorption in a line determines whether the line is classified weak or strong. This probability is characterized by the parameters $w=$ $\alpha_{0} /(\sigma+\kappa)$; here $\sigma$ is the continuum scattering coefficient, $\kappa$ is the continuum absorption coefficient, and $\alpha_{0}$ is the gaseous absorption coefficient at the center of a line. The quantity $w$ determines whether the core of a line will be nearly saturated or not.

A second parameter, $q=a_{0} / \kappa$, may also be formed from the same three fundamental coefficients. The role of the parameter $q$ - or even the basic fact that the theory contains two independent, fundamental parameters - does not seem to have been fully appreciated in the literature. It is predominantly $q$, the ratio of line absorption to continuum absorption, that determines, for weak lines as well as strong ones, the slope of the curve of growth - i.e., how the equivalent width varies with the lineabsorption coefficient. The competition between $\alpha_{0}$ and $\kappa$ for dominance in fixing the character of the curve of growth has no direct analogue in the simply reflecting model (i.e., an optically thin atmosphere with a diffusely reflecting ground). A physical explanation of the linear and square-root absorption laws, which emerge from the theory, may be obtained from a simplified discussion with random-walk theory of the mean number of scatterings.

The behavior of strong pressure-broadened lines is different in the central core, the outer wings, and the broad intermediate or transition region. The latter portion has no exact analogue in saturated lines in a simply reflecting atmosphere. All three regions of these strong lines follow the square-root absorption law, but for different reasons. The core and outer wings taken together are somewhat analogous to a pressurebroadened line in a simply reflecting atmosphere; but the extensive transition region is a unique result of multiple scattering in line formation. Numerical examples of

* Operated by the Association of Universities for Research in Astronomy, Inc., under contract with the National Science Foundation.

Sagan et al. (eds.), Planetary Atmospheres, 39-41.

All Rights Reseried. Copyright i 1971 by the I.A.L: 
curves of growth have been compared with the linear and square-root asymptotes for various continuum albedos.

In strong lines, a broad transition region, between the saturated core and the outer wings, absorbs roughly in proportion to $1 / \Delta \nu$ - or as the square root of the line absorption coefficient. This region has no analogue in ordinary, saturated Lorentz profiles. For weak continuum absorption the curve of growth continues to follow the squareroot absorption law as $w$ increases into the region of strong lines (where the central core is saturated), but the transition region dominates the equivalent width, except in the thin-crescent phase of the planet.

Line formation in finite but thick atmospheres may be treated with asymptotic formulae that express the emergent intensity in terms of Chandrasekhar's $H$ functions times a correction factor of the order of unity. A major problem in interpreting planetary spectra from a finite hazy atmosphere involves distinguishing between continuous absorption throughout the atmosphere and absorption at the ground. Intuitively one would expect the qualitative differences to be slight if the atmosphere is optically thick (say, $\tau \gtrsim 3$ ). Indeed, it is possible to construct a finite atmosphere of thickness $\tau_{0}$ and with continuum albedo $\tilde{\omega}_{c}=1$ that closely mimics the continuous and line absorption of a semi-infinite atmosphere with a lower continuum albedo, $\tilde{\omega}_{c}^{\prime}$. As we might have anticipated, the required finite thickness is $\tau_{0} \sim\left[3\left(1-\tilde{\omega}_{c}^{\prime}\right)\right]^{-1 / 2}$, the same as the mean depth of continuum absorption in the semi-infinite atmosphere. Another interesting result, which also could have been anticipated from the invariance principles, is that there is a ground albedo that will cause any thick but finite atmosphere to reflect the same intensity as a semi-infinite one having the same scattering albedo.

Phase variations of equivalent widths constitute one of the more important types of data for investigating hazy planets. These phase variations have been derived for several limiting cases and approximations. The use of a single point on the disk to represent the entire planet and thereby avoid a complicated averaging works moderately well.

The available information on the various $\mathrm{CO}_{2}$ absorption bands in Venus' spectra has been examined to ascertain whether a scattering model for the atmosphere can fit all the data without additional ad hoc assumptions. Some previous work has indicated apparent inconsistencies between theory and observations; however, a consistent model can be obtained, provided that the scattering albedo, $\tilde{\omega}_{c}$, is close to unity around $1.71 \mu$. Scattering albedos, for isotropic scattering, may be converted to equivalent values for other scattering functions with van de Hulst's similarity relations. A self-consistent scattering model is definitely indicated. The gaseous abundance 'above the cloud tops', although frequently mentioned in the literature, is a concept without meaning.

The visual albedos show that the atmosphere has an optical thickness of $\tau \gtrsim 20$, which is virtually infinite. The profile of the $1.05 \mu$ band gives a $\mathrm{CO}_{2}$ specific abundance (the amount in a column of unit cross section and a length of one photon mean free path) that is accurate to about a factor of two. The phase variation of the $8689 \AA$ band is consistent with what would be expected for this specific abundance and the measured continuum albedo in this region. However, the phase variation of the strong 
$1.6 \mu$ bands requires that the single-scattering albedo in the continuum, $\tilde{\omega}_{c}$, be very close to unity. Direct measurements of the planetary albedo indicate a somewhat smaller $\tilde{\omega}_{c}$, but the discrepancy is probably not serious. A third means of estimating the albedo in this region is provided by a moderately strong isotopic band at $1.71 \mu$; the strength of this band also seems to require a $\tilde{\omega}_{c}$ of essentially unity.

The detailed arguments leading to these conclusions are published elsewhere [1, 2].

\section{References}

[1] Chamberlain, J. W.: 1970, 'Behavior of Absorption Lines in a Hazy Planetary Atmosphere', Astrophys. J. 159, 137-158.

[2] Chamberlain, J. W. and Smith, G. R.: 1970, 'Interpretation of the Venus $\mathrm{CO}_{2}$ Absorption Bands', Astrophys. J. 160, 755-765. 